\title{
Novel surgical management of an extensive recurrent Hickman line thrombosis involving the SVC and right atrium
}

\author{
Anna Zingale ${ }^{1}$, Danai Karamanou ${ }^{1}$, Pietro Malvindi ${ }^{2}$, Suvitesh Luthra $^{1}$, and Sunil Ohri ${ }^{3}$ \\ ${ }^{1}$ Southampton University Hospitals NHS Trust \\ ${ }^{2}$ University Hospital Southampton NHS Foundation Trust \\ ${ }^{3}$ Southampton University Hospitals
}

July 19, 2021

\begin{abstract}
Intravenous central line catheters are often at risk of line-related thrombosis. We report on how the cardiopulmonary bypass strategy was tailored to the particular anatomical challenges with the use of an unconventional venous cannulation site to optimise intraoperative venous drainage, improve surgical exposure and avoid circulatory arrest in a case with a complex intracardiac thrombosis. This report also highlights the importance of assiduously monitoring the efficacy of anticoagulation therapy, especially in the context of small bowel syndrome.
\end{abstract}

\section{Title}

Novel surgical management of an extensive recurrent Hickman line thrombosis involving the SVC and right atrium

Authors:

Anna Zingale, MD1, Danai M. Karamanou, $\mathrm{MD}^{1}$, Pietro G. Malvindi, MD, $\mathrm{PhD}^{1}$, Suvitesh Luthra, $\mathrm{MS}^{1}$, Sunil K. Ohri, $\mathrm{MD}^{1}$

${ }^{1}$ Wessex Cardiothoracic Centre, University Hospital Southampton, Southampton, United Kingdom

Abstract

Intravenous central line catheters are often at risk of line-related thrombosis. We report on how the cardiopulmonary bypass strategy was tailored to the particular anatomical challenges with the use of an unconventional venous cannulation site to optimise intraoperative venous drainage, improve surgical exposure and avoid circulatory arrest in a case with a complex intracardiac thrombosis. This report also highlights the importance of assiduously monitoring the efficacy of anticoagulation therapy, especially in the context of small bowel syndrome.

\section{INTRODUCTION}

In vivo incidence of catheter-related right atrial thrombosis ranges between 5.4-12.5\%. However in postmortem examinations of patients with intravenous central line catheters this liability has been reported to be twice as high. Given that these patients are often asymptomatic this complication is commonly underdiagnosed. Depending on the size of the thrombus, rescue therapy may include surgical thrombectomy ${ }^{1}$.

We present an intriguing case requiring a unique surgical approach and challenging postoperative anticoagulative management. 


\section{CASE REPORT}

A 46-year-old lady underwent previous extensive small bowel excision for volvulitic ischaemia thereafter required long-term total parenteral nutrition therapy via a Hickman line. Her care was complicated by SVC thrombosis. Initial medical intervention included SVC stenting and anticoagulation with warfarin which was administered orally. Of note is the variable warfarin absorption in patients with short bowel syndrome $2^{2} 45$. In this case INR regulation was poor, leading to subcutaneous administration of therapeutic doses of enoxaparin sodium injections instead.

20 months after initial treatment, the patient re-presented with a further thrombus evident on transthoracic echocardiogram (figure 1C). The finding was confirmed with a venogram and cardiac MRI (figures 1A-1B). The thrombus extended from the distal aspect of the SVC stent into the right atrium, involving the entire chamber whilst abutting the tricuspid valve. An incidental finding of a patent foramen ovale was established, which increases the risk of embolic stroke.

The management of this case required a novel surgical approach.

Following median sternotomy and systemic heparinization, the patient was placed on total cardiopulmonary bypass with insertion of an arterial cannula in the ascending aorta and primary venous cannulation of the IVC. A second venous cannula, placed in the brachiocephalic vein, offered an alternative to a classic SVC cannulation ${ }^{6}$. This approach has been implemented in congenital and minimally invasive cardiac surgery. We opted for this approach to optimise upper-body drainage while maintaining myocardial and organ preservation, thereby avoiding the need for circulatory arrest. The patient was cooled down to $24^{\circ} \mathrm{C}$. After opening of the right atrium a vascular clamp was placed between the junction of the brachiocephalic vein and the SVC to reduce venous backflow. Residual venous drainage from the Azygous was dealt with by pump suction.

The complex thrombus extending from the SVC stent encompassing the Hickman line and extending into the right atrium, was excised. The PFO was identified and closed with a continuous suture. Thereafter the SVC stent was decorticated from the SVC wall. The Hickman line was trimmed, so that only $2-3 \mathrm{~cm}$ remained within the atrium and a pericardial patch was used to reconstruct the SVC before closure of the right atrium. The patient was readily weaned from cardiopulmonary bypass and closed routinely. Postoperative recovery was uncomplicated. Anticoagulation was resumed with an enoxaparin dose of $1.5 \mathrm{mg} / \mathrm{kg}$ once daily. We referred the patient to the haematology service for further investigations.

Unfortunately one year following surgery the patient presented with a 4-month history of dyspnoea and fatigue. A chest CT and confirmatory transoesophageal echocardiogram revealed a large right atrial thrombus once more abutting the tricuspid valve (Figures 2-3).

Haematological investigations were undertaken demonstrating antithrombin III activity within normal limits and no presence of heparin antibodies. She was commenced on intravenous unfractionated heparin infusion (maintaining APTR levels between 1.8 and 3) and proceeded to urgent redo surgery.

A redo-sternotomy was performed. Sharp dissection was required around the atrium, which was firmly adherent to the pericardium. Cardiopulmonary bypass was established via distal ascending aortic and bicaval cannulation, with direct SVC cannulation, superior to the previous patch repair. A right atriotomy was performed after snaring of the cavae. The chronic nature of the thrombus was reflective in the signs of encapsulation. Nonetheless a successful excision was achieved. Where the Hickman line tip abutted the lateral wall of the atrium it appeared abraded, with an adherent thrombus on its surface. Once more the Hickman line was therefore shortened. The operation was concluded in a routine fashion. Anticoagulation was resumed with an increased subcutaneous enoxaparin dose of $100 \mathrm{mg}$ twice daily (over $1 \mathrm{mg} / \mathrm{kg}$ twice daily) comparative to the preoperative dose of $120 \mathrm{mg}$ once daily.

The anti-factor Xa levels were monitored weekly for 1 month post-operatively to ensure adequate anticoagulation. Dose adjustments were implemented after checking anti-Xa levels. In addition the specialist intestinal failure team reviewed the case as part of an umbrella plan to trial oral nutrition with a long-term goal of 
removing the Hickman line. Unfortunately the patient was unable to tolerate oral nutrition and remains on total parenteral feeding. Follow-up at 12 months has found no evidence of recurrent atrial thrombus.

\section{DISCUSSION}

This case highlights the paramount necessity to closely monitor the efficacy of anticoagulation therapy, as current guidelines of standard doses of factor X-inhibitors may not prevent catheter-related thrombosis ${ }^{7}$.

We also suggest tailoring the cardiopulmonary bypass strategy to the particular anatomical challenges, with the use of an alternative venous cannulation to optimise venous drainage and improve surgical exposure during removal of an intracardiac thrombus.

\section{CONFLICT OF INTERESTS}

The authors declare that there are no conflict of interests.

\section{ETHICS STATEMENT}

Institutional IRB approval was waived for our case study and we have verbal consent of the patient for publication. No patient identifiers have been included in the manuscript.

\section{REFERENCES}

1. Tran M, Wilcox T, Tran PN. Cather-related right atrial thrombosis. The Journal of Vascular Access. 21(3):300-307, 25 Sep 2019.

2. Brophy DF, Ford SL, Crouch MA. Warfarin resistance in a patient with short bowel syndrome. Pharmacotherapy. 1998;18:646-649.

3. Sobieraj DM, Wang F, Kirton OC. Warfarin resistance after total gastrectomy and Roux-en-Y esophagojejunostomy. Pharmacotherapy. 2008;28:1537-1541.

4. Owens JP, Mirtallo JM, Murphy CC. Oral anticoagulation in patients with short-bowel syndrome. DICP. 1990;24:585-589.

5. Strong AT, Sharma G, Nor Hanipah Z, et al. Adjustments to warfarin dosing after gastric bypass and sleeve gastrectomy. Surg Obes Relat Dis. 2018;14:700-706.

6. Aazami MH, MD, Gholoobi A, Amini S, Abdollahi-Moghadam A, Soltani G. Left Brachiocephalic Vein Cannulation in Bicaval Venous Drainage Is Safe, Effective, and Technically Advantageous. Tex Heart Inst J. 2016 Apr; 43(2): 144-147.

7. Fallouh N, McGuirk HM, Flanders SA, et al. Peripherally inserted central catheter - associated deep vein thrombosis: a narrative review. Am J Med 2015; 128: 722-738.

8. Bishop L, Dougherty L, Bodenham A, et al. Guidelines on the insertion and management of central venous access devices in adults. Int J Lab Hematol 2007; 29: 261-278.

\section{FIGURE LEGENDS}

Figure 1 (A) Venogram with filling defect $*^{*}$ ) at the distal aspect of the SVC stent. (B) Cardiac MRI demonstrates SVC occluded with thrombus (*) that dangles into the atrium. (C) Transthoracic echocardiogram shows the right atrial mass $\left(^{*}\right)$ falling into the tricuspid valve orifice.

Figure 2 Chest computed tomography (A) sagittal, (B) coronal and (C) axial plane. The arrow indicates the catheter surrounded by the thrombus.

Figure 3 Transoesophageal echocardiogram (A) four chambers, (B) bicaval and (C) aortic valve short-axis view of the atrial thrombus $(*)$. 

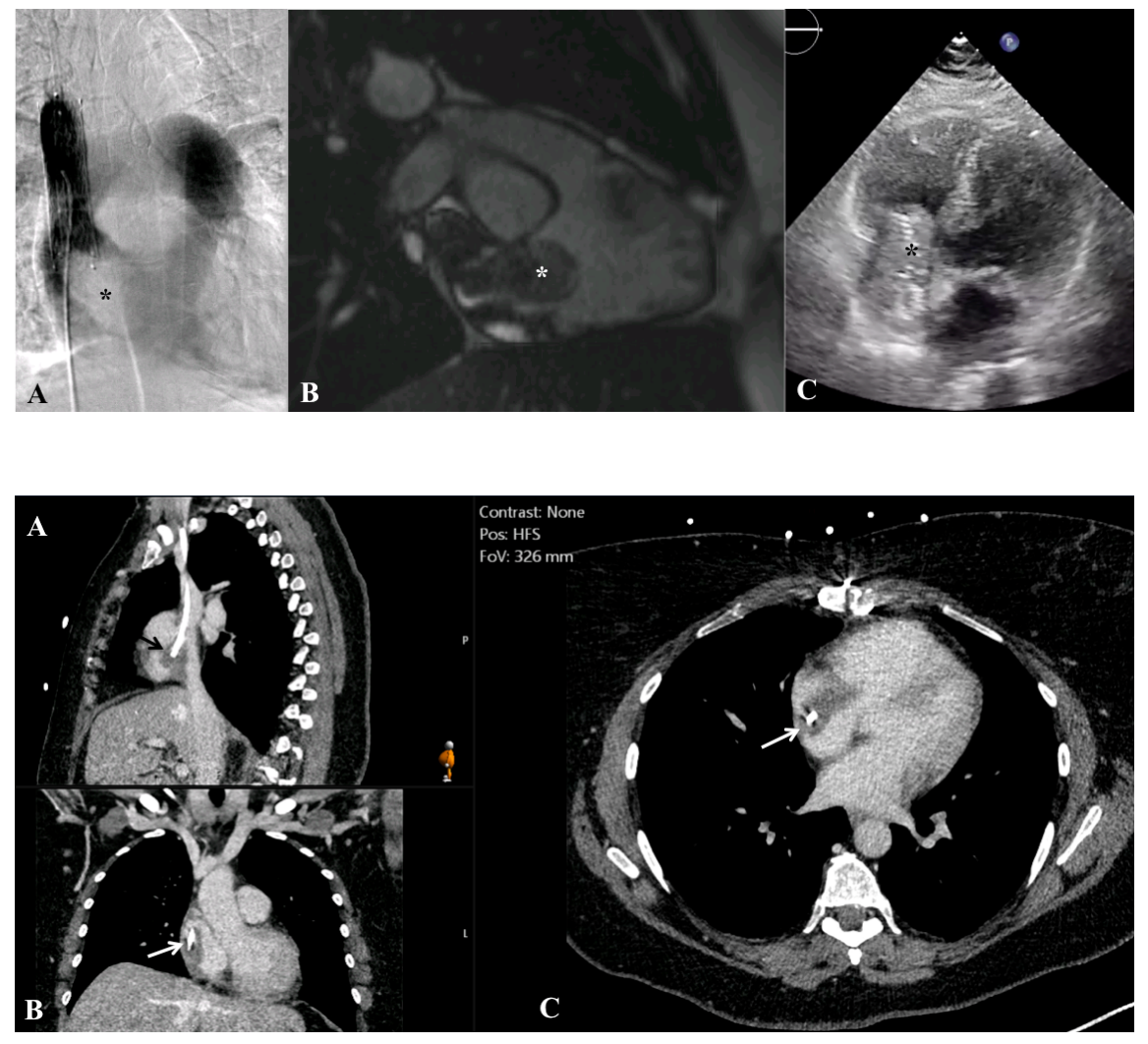


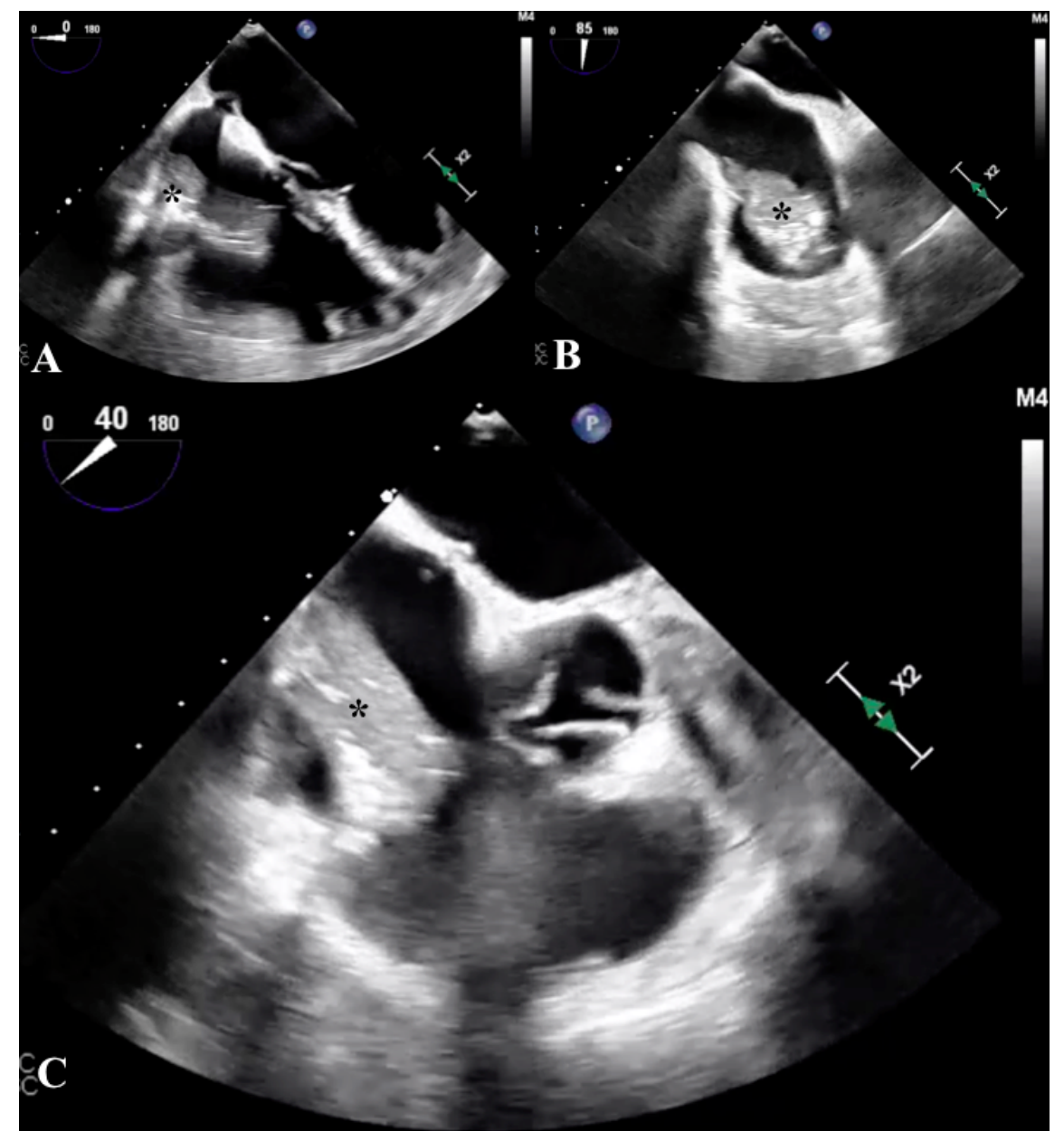

